\title{
A Novel Ferroptosis-Related IncRNA Signature and an Immune Perspective of Cervical Cancer
}

\section{Pengxiang Li}

Zhengzhou University First Affiliated Hospital

Dongchun Qin ( $\nabla$ qindongchun@zzu.edu.cn )

Zhengzhou University First Affiliated Hospital https://orcid.org/0000-0003-3564-1417

\section{Xuefeng LV}

Zhengzhou University First Affiliated Hospital

\section{Lu Liu}

Department of Clinical Laboratory, People's Hospital of Henan University of Chinese Medicine

\section{Mengle Peng}

Department of Clinical Laboratory, Henan No.3 Provincial People's Hospital

\section{Research}

Keywords: Cervical cancer, ferroptosis, IncRNA, qRT-PCR, WGCNA, immune checkpoint, TIDE, immunotherapy

Posted Date: September 22nd, 2021

DOl: https://doi.org/10.21203/rs.3.rs-900510/v1

License: (c) (i) This work is licensed under a Creative Commons Attribution 4.0 International License. Read Full License 


\section{Abstract}

Background: Cervical cancer (CC) is the most common reproductive neoplasm in women, especially in developing countries. Ferroptosis, a novel type of cell death, and IncRNAs play critical roles in the prognosis of CC patients and antitumor immunity.

Methods: A ferroptosis-related IncRNA signature (FRLS) was constructed by LASSO Cox regression analysis. Kaplan-Meier analysis, receiver operating characteristic (ROC) curve analysis, multivariate analysis, and nomogram were used to evaluate and predict the FRLS. Based on the FRLS, immune-related genes, the tumor microenvironment (TME), immune checkpoints, and immunotherapy were investigated.

Results: The FRLS was composed of ten IncRNAs and was markedly associated with the overall survival (OS) of CC patients. Gene set enrichment analysis (GSEA) demonstrated that the FRLS was largely associated with immune-related pathways. Weighted gene co-expression network analysis (WGCNA) was performed to analyze immune-related genes and to identify the optimal modules and genes. TLR4 was eventually identified, and its expression was verified in the Gene Expression Omnibus (GEO) database. Then, quantitative real-time PCR (qRT-PCR) was used to validate the results in CC and paracancerous tissues. Besides, our results showed that CD8+ T cells were significantly correlated between the low- and high-risk groups, and it could modulate ferroptosis during tumor immunotherapy. The expression of immune checkpoints was substantially different between the two groups. Additionally, tumor immune dysfunction and exclusion (TIDE) was applied to predict the sensitivity of immune checkpoint inhibitor (ICl) treatment.

Conclusion: The FRLS established was significantly associated with prognosis; moreover, the FRLS is a prospective therapeutic target, and combined with immunotherapy, can be used in the treatment of CC patients.

\section{Background}

Cervical cancer (CC) is one of the most prevalent malignancies in women and has become a global health crisis(1). Although CC can be prevented and cured if the disease is discovered in a timely manner, 604,127 people worldwide were affected by CC in 2020(2). Human papillomavirus (HPV) infection is considered the primary causative factor of CC, and more than $90 \%$ of CC patients are infected with highrisk HPV(3). In recent years, many studies have shown that CC was occurring at an increasingly young age(4). Few diseases showed global inequities as much as CC; one example is that the incidence of CC in rural areas is higher than that in urban areas, and the incidence in developing countries is higher than that in developed countries(5). A great deal of CC patients in the developing world, such as China, are diagnosed at an advanced stage due to low awareness of CC screening, and thus, traditional remedies (surgery, chemotherapy, and radiotherapy) for CC patients have shown minimal results. Therefore, the development of novel prognostic biomarkers and treatment options is urgent. 
Recently, a novel form of cell death known as ferroptosis, was discovered(6). Ferroptosis is associated with reactive oxygen species (ROS) and induces a characteristic iron-dependent form of cell death that is different from apoptosis and is associated with the development and therapeutic response of various cancer types. Increasing evidence indicates that ferroptosis is an adaptive process that is crucial to the elimination of tumor cells(7). Ferroptosis can induce lipid peroxidation (LPO) in cancer cells, and investigation of the responsiveness and adaptation of immune cells to LPO in the tumor microenvironment (TME) can further reveal the role of ferroptosis in tumor immunity. A previous study showed that ferroptosis was involved in T cell-mediated immunity and cancer immunotherapy. For example, CD8 + T cells triggered by immunotherapy can increase LPO in cancer cells, and the advancement of ferroptosis can boost the antitumor efficacy of immunotherapy. Immunotherapy has focused on the treatment of a variety of cancers(8). Immunotherapy based on cytotoxic T-lymphocyteassociated antigen 4 (CTLA-4) or programmed cell death protein 1 (PD1) has significant potential in the treatment of $\mathrm{CC}(9)$. It has been shown that immune cells in the TME, such as T and DC cells, participate in metabolic and growth signaling pathways similar to those of cancer cells(10). Consequently, ferroptosis inducers can also influence the activity and functions of immune cells by causing lipid peroxidation.

Long noncoding RNAs (IncRNAs), which are more than two hundred nucleotides in length, are noncoding RNAs that play a crucial role in molecular processes and the development of diseases, including tumors $(11,12)$. Increasing numbers of researchers are committed to analyzing the critical factors of IncRNAs that modulate the incidence and development of tumors. LncRNA PTENP1 inhibits cell proliferation, apoptosis, and epithelial-mesenchymal transition (EMT) by competitively binding to miR106b in CC(13). BBOX1-AS1 was found to promote CC progression by upregulating HOXC6 expression via miR-361-3p and HuR(14). GATA3-AS1 can maintain the expression of programmed cell death ligand 1 (PD-L1) protein through deubiquitination and can destabilize GATA3 protein by ubiquitination; this facilitates tumor development and immune evasion in triple-negative breast cancer (TNBC), which represents a novel method for the treatment of TNBC(15). A recent study showed that IncRNA LINC00618 promotes apoptosis and ferroptosis and that LINC00618 promotes a form of apoptosis-dependent ferroptosis in human leukemia cells(16). Recently, ferroptosis-related IncRNA studies of several cancers were performed(17-19), shuch as breast cancer, grastic cancer, Head and neck squamous cell carcinoma. However, no similar data have been reported on a ferroptosis-related IncRNA prognostic model of CC.

For this study, we acquired RNA-seq data of CC patients from The Cancer Genome Atlas (TCGA) database and the Gene Expression Omnibus (GEO) database, and ferroptosis genes were downloaded from the FerrDb database. Then, the FRLS was established through LASSO Cox regression to predict the prognosis of patients with CC. Furthermore, we evaluated the clinicopathological characteristics of the FRLS and the pathways involved in the FRLS. Subsequently, the combination of the FRLS and immunity, including immune-related genes, the TME, and immunotherapy, was investigated. Furthermore, Weighted gene coexpression network analysis (WGCNA) was used to identify optimal immune genes, CIBERSORT and ESTIMATE were used to evaluate immune cells in the TME, and tumor immune dysfunction and exclusion 
(TIDE) was performed to test the sensitivity of treatment with ICI. TLR4, which was identified via WGCNA, was validated in the CC tissues and normal samples.

\section{Results}

\subsection{Construction of the FRLS for prognosis}

In all, 512 ferroptosis-related IncRNAs were discovered by Pearson correlation analysis, and 15 IncRNAs were found through univariate Cox regression. Furthermore, 10 IncRNAs (MIR100HG, WDR86-AS1, AC131159.1, AC092171.2, AL021707.6, AC007998.3, AC024270.4, AC004847.1, AC243829.4, MIAT) were screened by LASSO and multivariate Cox regression (Fig. 1a, 1b), which indicates that they might be independent prognostic predictors of CC (Fig. 1c, 1d). The risk score was determined by the equation above, which was obtained by LASSO Cox regression.

\subsection{Validation of the FRLS in CC patients}

According to the cutoff on the ROC curve, as determined by the "survminer" package, the 273 CC patients were stratified into two groups: the low-risk $(n=56)$ and high-risk $(n=217)$ groups. The Kaplan-Meier (KM) curve showed that the patients in the low-risk group exhibited longer survival than those in the highrisk group (Fig. 2a). The heatmap showed the relative expression of 10 ferroptosis-related IncRNAs between low- and high-risk groups (Figure $2 \mathrm{~b}$ ). The area under the cure (AUC) predictive value of the FRLS was assessed by a time-independent ROC curve; the AUC was 0.838 at 1 year, 0.800 at 3 years, and 0.822 at 5 years (Fig. 2d). The risk plot showed an inverse relationship between the survival of patients with CC and the risk score: as the risk score increased, patient survival decreased (Fig. 2e, 2f). It is feasible that the FRLS is a good index that can be used to measure the prognosis of CC, as shown by the outcomes above.

\subsection{Correlations between the FRLS and the clinicopathological characteristics of CC}

A heatmap was used to demonstrate the relationship between the high- and low-risk groups and clinicopathological characteristics ( $T, M, N$, tumor stage, age, and grade). We found that $T(<0.001), N(<$ 0.01), and stage (<0.05) were significantly associated with the FRLS (Fig. 3a). The AUC of the multifactor ROC curve was 0.838 , which was higher than that of the other risk factors alone, indicating that the risk score performed better than the other clinicopathological characteristics (Fig. 3b). The results demonstrated that the risk score of the FRLS could be considered an independent predictor of the OS of CC patients. In addition, we analyzed the divergences in OS between the low- and high-risk groups classified according to clinicopathologic features. According to the subgroups classified by age, stage, grade, $\mathrm{T}$ stage, $\mathrm{M}$ stage, and $\mathrm{N}$ stage, the OS of the high-risk group was still significantly lower than that of the low-risk group (Supplementary Figure S1). 


\subsection{Enrichment Analysis and Gene set enrichment analysis (GSEA)}

Kyoto Encyclopedia of Genes and Genomes (KEGG) enrichment analysis was used to analyze the signaling pathways of differentially expressed ferroptosis genes, which are mainly involved in ferroptosis, PD-L1 expression, and the PD-1 checkpoint pathway in cancer (Fig. 4a). Molecular function (MF), biological processes (BP), and cellular components (CC) were analyzed through Gene Ontology (GO) enrichment of ferroptosis genes (Fig. 4b). GSEA(20) revealed that the FRLS was significantly enriched in immune-associated pathways, such as T-cell receptor signaling pathways, natural killer cell-mediated cytotoxicity, and cell adhesion molecules (CAMs) (Fig. 4c).

\subsection{Principal-component analysis (PCA)}

According to the all the IncRNAs (Fig. 5a), the 512 ferroptosis-related IncRNAs (Fig. 5b), and the FRLS (Fig. 5c), PCA(21) was performed to examine the discrepancies between the high- and low-risk groups. The distributions between the low- and high-risk groups were quite diverse, which indicates that the FRLS can be used to distinguish these two groups.

\subsection{Independence of the prognostic ability of the FRLS}

Univariate and multivariate Cox regression analyses were performed to evaluate whether the risk score was an independent predictive characteristic of CC. The hazard ratio (HR) and $95 \%$ confidence interval $(\mathrm{Cl})$ for the risk scores were 1.031 and 1.008-1.054 ( $<<0.007)$, respectively, which were analyzed by univariate Cox regression (Fig. 6a). In the multivariate Cox regression analysis, the HR was 1.025 with a 95\% $\mathrm{Cl}$ of 1.007-1.042 (Fig. 6b), which demonstrates that the risk score can be an independent risk factor again. The nomogram consisted of risk classes and risk factors and was applied to predict the incidence of OS at 1,3, and 5 years (Fig. 6c). A nomogram including risk factors (age, grade, stage) was constructed and predicted the 1-, 3-, and 5-year OS.

\subsection{Identification of the optimal module via WGCNA}

Overall, 2483 immune-related genes were analyzed via the WGCNA algorithm. Initially, according to the Rsquared value of 0.85 , a soft threshold of 3 was acquired to construct a scale-free network (Fig. 7a). These genes were divided into five modules (blue, yellow, turquoise, brown, and gray modules), where the gray module indicated that the genes were not assigned to any module (Fig. 7b). Then, the correlation between these five gene modules and risk was investigated. Of these modules, the turquoise module was the most significant one (Fig. 7c). And in this module, the relationship between gene significance (GS) and the module members was greatly associated with the low-risk group, whose correlation was 0.51 (Fig. 7d).

\subsection{Identification and verification of TLR4}


The Venn diagram illustrates seven genes that were obtained from the intersection of the turquoise module genes, which included 249 genes, and 259 ferroptosis genes (Fig. 8a). The seven genes were analyzed for differences based on the filter criteria of $p<0.05$, $\log F C>2$, finally, TLR4 was identified. Then, the GEO databases were used to verify the expression of TLR4, which was significantly higher in CC than that in normal tissues. Besides, the results of qRT-PCR was consistent with the GEO databases (Fig. 8C). Furthermore, the protein expression of TRL4 in CC tissues was verified by using Human Protein Atlas (HPA) database (Fig. 8d,e).

\subsection{Estimation of the TME and immune checkpoint inhibitor therapy}

CIBERSORT(22) was used to obtain immune cells and their proportions in CC from the gene expression matrix. The R package ESTIMATE(23) was used to acquire the scores of immune cells and stromal cells. An immune heatmap based on multiple immune algorithms (e.g., TIMER, CIBERSORT, EPIC) showed differences in immune cells between the high- and low-risk groups (Fig. 9a). The immune score based on the FRLS was investigated and was highly significantly different $(P=1.3 e-05)$ between the high- and low-risk groups (Fig. 9b). The relationship between the FRLS and the expression of immune checkpoint proteins was analyzed because of the prominence of ICl-based immunotherapy. A remarkable difference was observed in the expression of immune checkpoint proteins(24), such as PDCD-1 (PD-1) and CTLA4, between the low- and high-risk groups (Fig. 9c). Furthermore, patients in the low-risk group had higher TIDE scores than those in the high-risk group, which demonstrates that patients in the high-risk group were more susceptible to $\mathrm{ICl}$ treatment than their low-risk counterparts (Fig. 9d).

CD4 memory resting T cells (Fig. 10a) and Macrophages M0 (Fig. 10b) demonstrated a significant positive correlation with the risk scores. In contrast, activated T-cell CD4 memory activation (Fig. 10c) and CD8 + T cells (Fig. 10d) were negatively correlated with the risk score. For each patient, single sample GSEA (sSGSEA) was used to obtain the immune enrichment scores of the diverse immune cells. Then, we analyzed the relationship between the scores and the FRLS (Fig. 10e). Unsurprisingly, immune cells (e.g., T helper cells and CD8 + T cells) and immune function (e.g., checkpoint, cytolytic activity, $T$ cell costimulation) were significantly different between the low- and high-risk groups (Fig. 10f).

\section{Discussion}

Cervical cancer, as the fourth most common tumor type in women, is a major threat to women's health worldwide. However, the molecular mechanisms underlying the development of cervical cancer are not yet fully understood. Emerging evidence has shown the potential of inducing ferroptosis as a cancer therapy, particularly for eliminating tumors that are tolerant to traditional radiotherapy and chemotherapy. Increasing numbers of studies have shown that IncRNAs are related to a variety of diseases(25-27), such

as diabetic cardiomyopathy, liver cancer, CC, and atherosclerosis. Interestingly, some IncRNAs and microRNAs are largely considered key mediators that modulate ferroptosis. Therefore, we developed a 
model of ferroptosis-related IncRNAs that can be used as a novel biomarker in the treatment of CC patients.

In this study, the FRLS, comprising 10 IncRNAs (MIR100HG, WDR86-AS1, AC131159.1, AC092171.2, AL021707.6, AC007998.3, AC024270.4, AC004847.1, AC243829.4, MIAT), was constructed to predict OS in CC patients. For MIR100HG, we found that MIR100HG sponged miR-5590-3p to promote the progression of TNBC, thereby upregulating OTX1, which may be a novel biomarker of TNBC(28). A recent study showed that in gastric cancer, CXXC finger protein 4 (CXXC4) is involved in the ELK1/MIR100HG pathway to inhibit the immune evasion of tumors by restraining the CDK18-ERK1/2 axis(29). One study showed that the expression of MIR100HG and miR-204-5p had a marked relationship and that MIR100HG promoted tumor cell progression by downregulating miR-204-5p in laryngeal squamous cell carcinoma(30). MIAT, as a competing endogenous RNA that sponges miR-22-3p, upregulated DAPK2, which resulted in cardiomyocyte apoptosis(26). Research has demonstrated that MIAT can sponge miR214 to facilitate migration and invasiveness of hepatocellular carcinoma cells and that MIAT can be a novel marker used in the treatment of HCC patients(31). As reported previously, a ceRNA network exists between MIAT and miR-186, and MIAT sponged miR-186-3p to regulate VEGFC, which facilitated the proliferation, invasiveness, and migration of osteosarcoma cells(32). Another study reported AL021707.6 as a prospective crucial moderator of apoptosis in polymorphonuclear leukocytes(33). Other IncRNAs have not yet been studied and will be further explored in future studies.

Furthermore, the KEGG analysis showed that the differentially expressed genes were mainly involved in the ferroptosis pathway, HIF-1 signaling pathway, PD-L1 expression, autophagy pathway, and the PD-1 checkpoint pathway in cancer and had a relationship with immunotherapy and ferroptosis. According to the risk score, CC patients were classified into two groups; of these groups, the low-risk group had better clinical outcomes. The multifactor ROC curve showed that the risk score can be an independent factor and superior to other traditional clinical characteristics in predicting OS. A nomogram was constructed to show the predictors of the 1-, 3-, and 5-year OS. Overall, the risk model of ferroptosis-related IncRNAs was effective and accurate for predicting the prognosis of patients. In this study, we also found that the risk score could increase with tumor progression. Moreover, during the treatment process, the pathological stage of cancer was the critical factor related to the OS of CC patients. However, the prognosis of patients at the same pathological stage was quite distinctive, which indicates that the current staging system is not comprehensive enough to reflect the heterogeneity of patients. Therefore, we established the FRLS to complement the staging system and to explore a novel biomarker to predict the prognosis of CC patients. Additionally, we validated the FRLS through several methods, and thus, this signature is an effective prognostic model.

Some genes have been confirmed to influence CC due to their abilities to modulate disease occurrence and development as well as immune response, even immunotherapy. WGCNA was used to analyze the immune-related genes, and subsequent selections eventually identified TLR4. Nanosystems activated by TLR4 have been shown to trigger the production of high levels of proinflammatory cytokines and chemokines by macrophages and DCs during immunotherapy(34). Moreover, TLR4 was also shown to be 
associated with ferroptosis; for example, suppression of TLR4 alleviates oxidative stress-induced injury and reduces ferroptosis activation in hippocampal neurons(35).

The TME of CC comprises various immune and stromal cells and likely modulates several hallmarks of tumors, including proliferation, invasion, and chemotherapy resistance(36). Some studies have shown that ferroptosis can occur in immune cells in the TME and as a result of the interaction between immune cells and tumor cells, which offers the possibility of targeting ferroptosis in immunotherapy(10). Our study demonstrated that the proportions of Macrophages M0, T cells CD8, B cells memory were related to the risk model, which exhibited a notable correlation with the risk score. A previous study showed that the role of CD8 + T cells was strengthened by cancer immunotherapy $(37,38)$. An interaction was observed between immunotherapy-activated CD $8+T$ cells and ferroptosis in which CD $8+T$ cells could increase ROS and ferroptosis, which is conducive to antitumor efficiency of immunotherapy(8). Furthermore, the effectiveness of radiation treatment in vivo depends on the existence of CD8 + T cells $(39,40)$, which regulate cancer ferroptosis via IFNy. One study showed that ferroptosis inducers or blockers can affect the antineoplastic immunity mediated by T cells; examples are the ferroptosis blocker ferrostatin-1, which prevents T cell-mediated specific killing, and GPX4 blockers, which selectively kill CD8 + T cells to promote tumor cell growth(41).

In our study, we explored the relationship between ICls and the FRLS. Over the past several years, substantial progress has been made in the development of ICls for tumor immunotherapy. Moreover, ferroptosis coupled with ICls can improve anticancer efficacy(42), and TIDE scores have revealed the enormous potential of $\mathrm{ICl}$ treatment. A recent study found that TYRO3 can restrain cancer cells, induce ferroptosis stimulated by anti-PD-1/PD-L1 treatment, and remodel the TME, leading to resistance to antiPD-1/PD-L1 treatment(43). This might be an effective way to combine ferroptosis and immunotherapy based on ICls (PD-1, CTLA-4) to treat CC patients.

However, this study still has some limitations. First, the number of CC samples in the TCGA database was comparatively small. Second, because of the lack of clinical samples, the clinical reliability and prognostic stability of the FRLS cannot be completely ensured. The mechanism of ferroptosis-related IncRNAs will be validated in later studies, which will provide more convincing data for clinical treatment.

\section{Conclusion}

In summary, we established the FRLS to predict the OS of patients with CC, and this signature could provide an approach to discover the molecular mechanism of ferroptosis-related IncRNAs. Furthermore, TLR4, which is involved in the occurrence of ferroptosis and associated with immunotherapy, was validated by qRT-PCR in CC and normal tissues. In addition, the combination of ferroptosis-related IncRNAs and tumor immunity, such as immunotherapy, will be a more effective treatment for CC patients.

\section{Materials And Methods}




\subsection{Data acquisition}

CC RNA-seq and corresponding clinical data were obtained from the TCGA database (https://portal.gdc.cancer.gov/), which contained 306 CC cases and 3 normal tissue samples. In all, 274 patients with more than 30 days of follow-up data were enrolled. The GSE9750, GSE44001, and GSE63514 datasets were downloaded from the GEO database (https://www.ncbi.nlm.nih.gov/geo/) to validate gene expression. The ferroptosis genes were downloaded from the FerrDb database(44) (http://www.zhounan.org/ferrdb/ ), which was the first database to contain genes that regulate ferroptosis and diseases related to ferroptosis. Finally, the 259 ferroptosis genes obtained from the FerrDb database consisted of 108 drivers, 69 suppressors, and 111 markers. In all, 2483 immune-related genes were acquired from ImmPort (https://www.immport.org). CC tissue samples were obtained from the First Affiliated Hospital of Zhengzhou University, and consent was granted by all patients. This study was also authorized by our hospital ethics committee (Ethics No. 2018-KY-28).

\subsection{Construction and validation of the FRLS for CC}

In all, 259 ferroptosis genes and 13,162 IncRNAs were analyzed by Pearson correlation analysis to identify 512 ferroptosis-related IncRNAs; the standard correlation coefficients were $|R|>0.4$ and $P<0.001$. Then, a univariate Cox regression analysis and a LASSO Cox regression analysis were used to establish a prognostic model of $\operatorname{IncRNAs}(45,46)$. The risk score was determined by the equation: risk score $=\sum$ coeffi IncRNA* exp IncRNA, where coeffi IncRNA was the coefficient of IncRNA and exp IncRNA was the expression of IncRNA. The patients with CC were classified into low- and high-risk groups based on the cutoff value of the ROC curve.

\subsection{Principal component analysis and enrichment analysis}

PCA was used for dimension reduction and to effectively visualize the high-dimensionality data. The limma package was used to screen the differentially expressed ferroptosis genes; these genes were analyzed by GO and KEGG(47) analyses, for which the clusterProfiler R package was used. The cutoff criterion was $p<0.05$.

\subsection{Predictive nomogram and independence analysis of the FRLS}

A nomogram was established to integrate the risk elements to predict the 1-, 3-, and 5-year OS of CC patients. Multivariate and univariate Cox regression analyses were applied to decide whether the risk score could be an independent element of prognosis.

\subsection{Analysis of immune genes by WGCNA}

WGCNA $(48,49)$ is a method of classifying highly correlated genes into the same module and then linking the module genes to the sample traits to evaluate GS. The WGCNA package was used to identify modules of interest from the immune genes. The module trait showed the correlation of eigengenes and risk by 
Pearson analysis, which selected the optimal module (turquoise module). Furthermore, the desired genes were further validated in the GEO database.

\subsection{Validation of TLR4 expression by qRT-PCR}

TRIzol reagent was used to isolate total RNA from the tissue samples (16 CC samples and16 paracancerous tissues), and then a cDNA kit was used to reverse transcribe the total RNA to cDNA. Finally, the relative mRNA expression of TLR4, which was determined using the $2-\Delta \Delta C T$ method, was detected and compared between cancer tissues and paracancerous tissues. The protein expression levels of TLR4 in human normal and CC tissues were estimated in the Human Protein Atlas (HPA) (http://www.proteinatlas.org).

\subsection{Immune analysis}

The CIBERSORT algorithm was used to extract the immune cells and their relative levels from the gene expression matrix after which they were scored by ESTIMATE. To obtain an immunological status analysis of every patient in the TCGA-CC cohort, the relative levels of 29 immune cell types in the TME(50) were determined by the R package "GSVA" via ssGSEA(51) algorithm. The immune gene set was downloaded from the Gene Set Enrichment Analysis (https://www.gsea-msigdb.org/gsea/msigdb/). The correlation between immune cells and the risk score was also analyzed. TIDE(52) was used to predict the effect of ICl therapy.

\subsection{Statistical analysis}

The analyses were performed using R software 4.0.3 and GraphPad Prism 9. Batch effects between three GEO datasets were corrected with the sva package. Kaplan-Meier survival analysis and log-rank test were applied to differentiate the OS between the two groups. For each analysis, a P value less than 0.05 was considered statistically significant.

\section{Abbreviations}

CC

cervical cancer

FRLS

ferroptosis-related IncRNA signature

ROC

receiver operating characteristic

TME

tumor microenvironment

OS

overall survival

GSEA

gene set enrichment analysis 
WGCNA

weighted gene co-expression network analysis

GEO

Gene Expression Omnibus

qRT-PCR

quantitative real-time PCR

TIDE

tumor immune dysfunction and exclusion

ICl

immune checkpoint inhibitor

HPV

Human papillomavirus

ROS

reactive oxygen species

LPO

lipid peroxidation

CTLA-4

cytotoxic T-lymphocyte-associated antigen 4

PD1

programmed cell death protein 1

IncRNAs

long noncoding RNAs

EMT

epithelial-mesenchymal transition

PD-L1

programmed cell death ligand 1

TNBC

triple-negative breast cancer

TCGA

The Cancer Genome Atlas

MF

molecular function

BP

biological processes

CC

cellular components

CAMs

cell adhesion molecules

KM

Kaplan-Meier 
AUC

area under the cure

PCA

principal-component analysis

HR

hazard ratio

$\mathrm{Cl}$

confidence interval

\section{Declarations}

Availability of data and materials

The raw data of this study are derived from the TCGA database (https://portal.gdc.cancer.gov/), GEO database(https://www.ncbi.nlm.nih.gov/geo/), and FerrDb database (http://www.zhounan.org/ferrdb/ ), which are publicly available databases.

Acknowledgements

We were grateful to the Cancer Genome Atlas (TCGA) database, the Gene Expression Omnibus (GEO) database, FerrDb database and ImmPort database, which made the data available.

Funding

This study was funded by grants from the Scientific and Technological Project of Henan Province (172102310077). Funds for Creative Research Team of Henan Province.

Contributions

This research was conducted in collaboration with all authors. PXL and XFL Collated and analyzed data. PXL drafted the paper. LL and MLP offered constructive advice. All authors read and approved the final manuscript.

Ethics approval and consent to participate

This study was carried out in accordance with the hospital ethics committee of the First Affiliated Hospital of Zhengzhou University

Consent for publication

Not applicable

Competing interests

The authors declare that they have no competing interests. 


\section{References}

1. Bray F, Ferlay J, Soerjomataram I, Siegel RL, Torre LA, Jemal A. Global cancer statistics 2018: GLOBOCAN estimates of incidence and mortality worldwide for 36 cancers in 185 countries. Cancer J Clin. 2018;68(6):394-424.

2. Delman KA. Introducing the "Virtual Tumor Board" series in CA: A Cancer Journal for Clinicians. Cancer J Clin. 2020;70(2):77.

3. Walboomers JM, Jacobs MV, Manos MM, Bosch FX, Kummer JA, Shah KV, et al. Human papillomavirus is a necessary cause of invasive cervical cancer worldwide. J Pathol. 1999;189(1):12-9.

4. Chen W, Zheng R, Zhang S, Zeng H, Xia C, Zuo T, et al. Cancer incidence and mortality in China, 2013. Cancer letters. 2017;401:63-71.

5. Chen W, Zheng R, Baade PD, Zhang S, Zeng H, Bray F, et al. Cancer statistics in China, 2015. Cancer J Clin. 2016;66(2):115-32.

6. Dixon SJ, Lemberg KM, Lamprecht MR, Skouta R, Zaitsev EM, Gleason CE, et al. Ferroptosis: an irondependent form of nonapoptotic cell death. Cell. 2012;149(5):1060-72.

7. Dixon SJ. Ferroptosis: bug or feature? Immunological reviews. 2017;277(1):150-7.

8. Wang W, Green M, Choi JE, Gijón M, Kennedy PD, Johnson JK, et al. CD8(+) T cells regulate tumour ferroptosis during cancer immunotherapy. Nature. 2019;569(7755):270-4.

9. Chitsike L, Duerksen-Hughes P. The Potential of Immune Checkpoint Blockade in Cervical Cancer: Can Combinatorial Regimens Maximize Response? A Review of the Literature. Curr Treat Options Oncol. 2020;21(12):95.

10. Xu H, Ye D, Ren M, Zhang H, Bi F. Ferroptosis in the tumor microenvironment: perspectives for immunotherapy. Trends in molecular medicine. 2021.

11. Brown CJ, Hendrich BD, Rupert JL, Lafrenière RG, Xing Y, Lawrence J, et al. The human XIST gene: analysis of a $17 \mathrm{~kb}$ inactive X-specific RNA that contains conserved repeats and is highly localized within the nucleus. Cell. 1992;71(3):527-42.

12. Bartolomei MS, Zemel S, Tilghman SM. Parental imprinting of the mouse $\mathrm{H} 19$ gene. Nature. 1991;351(6322):153-5.

13. Fan Y, Sheng W, Meng Y, Cao Y, Li R. LncRNA PTENP1 inhibits cervical cancer progression by suppressing miR-106b. Artif Cells Nanomed Biotechnol. 2020;48(1):393-407.

14. Xu J, Yang B, Wang L, Zhu Y, Zhu X, Xia Z, et al. LncRNA BBOX1-AS1 upregulates HOXC6 expression through miR-361-3p and HuR to drive cervical cancer progression. Cell proliferation. 2020;53(7):e12823.

15. Zhang M, Wang N, Song P, Fu Y, Ren Y, Li Z, et al. LncRNA GATA3-AS1 facilitates tumour progression and immune escape in triple-negative breast cancer through destabilization of GATA3 but stabilization of PD-L1. Cell proliferation. 2020;53(9):e12855. 
16. Wang Z, Chen X, Liu N, Shi Y, Liu Y, Ouyang L, et al. A Nuclear Long Non-Coding RNA LINC00618 Accelerates Ferroptosis in a Manner Dependent upon Apoptosis. Mol Ther. 2021;29(1):263-74.

17. Zhang K, Ping L, Du T, Liang G, Huang Y, Li Z, et al. A Ferroptosis-Related IncRNAs Signature Predicts Prognosis and Immune Microenvironment for Breast Cancer. Frontiers in molecular biosciences. 2021;8:678877.

18. Pan J, Zhang X, Fang X, Xin Z. Construction on of a Ferroptosis-Related IncRNA-Based Model to Improve the Prognostic Evaluation of Gastric Cancer Patients Based on Bioinformatics. Frontiers in genetics. 2021;12:739470.

19. Tang Y, Li C, Zhang YJ, Wu ZH. Ferroptosis-Related Long Non-Coding RNA signature predicts the prognosis of Head and neck squamous cell carcinoma. Int J Biol Sci. 2021;17(3):702-11.

20. Damian D, Gorfine M. Statistical concerns about the GSEA procedure. Nat Genet. 2004;36(7):663. author reply.

21. Mi JX, Zhang YN, Lai Z, Li W, Zhou L, Zhong F. Principal Component Analysis based on Nuclear norm Minimization. Neural networks: the official journal of the International Neural Network Society. 2019;118:1-16.

22. Newman AM, Liu CL, Green MR, Gentles AJ, Feng W, Xu Y, et al. Robust enumeration of cell subsets from tissue expression profiles. Nature methods. 2015;12(5):453-7.

23. Yoshihara K, Shahmoradgoli M, Martínez E, Vegesna R, Kim H, Torres-Garcia W, et al. Inferring tumour purity and stromal and immune cell admixture from expression data. Nature communications. 2013;4:2612.

24. Sharma P, Allison JP. The future of immune checkpoint therapy. Science. 2015;348(6230):56-61.

25. Ding X, Jia X, Wang C, Xu J, Gao SJ, Lu C. A DHX9-IncRNA-MDM2 interaction regulates cell invasion and angiogenesis of cervical cancer. Cell death differentiation. 2019;26(9):1750-65.

26. Zhou X, Zhang W, Jin M, Chen J, Xu W, Kong X. IncRNA MIAT functions as a competing endogenous RNA to upregulate DAPK2 by sponging miR-22-3p in diabetic cardiomyopathy. Cell Death Dis. 2017;8(7):e2929.

27. Zhang Z, Wang S, Liu Y, Meng Z, Chen F. Low IncRNA ZNF385D-AS2 expression and its prognostic significance in liver cancer. Oncol Rep. 2019;42(3):1110-24.

28. Chen FY, Zhou ZY, Zhang KJ, Pang J, Wang SM. Long non-coding RNA MIR100HG promotes the migration, invasion and proliferation of triple-negative breast cancer cells by targeting the miR-55903p/OTX1 axis. Cancer Cell Int. 2020;20:508.

29. Li P, Ge D, Li P, Hu F, Chu J, Chen X, et al. CXXC finger protein 4 inhibits the CDK18-ERK1/2 axis to suppress the immune escape of gastric cancer cells with involvement of ELK1/MIR100HG pathway. J Cell Mol Med. 2020;24(17):10151-65.

30. Huang Y, Zhang C, Zhou Y. LncRNA MIR100HG promotes cancer cell proliferation, migration and invasion in laryngeal squamous cell carcinoma through the downregulation of miR-204-5p. Onco Targets Ther. 2019;12:2967-73. 
31. Huang X, Gao Y, Qin J, Lu S. IncRNA MIAT promotes proliferation and invasion of HCC cells via sponging miR-214. American journal of physiology Gastrointestinal liver physiology. 2018;314(5):G559-g65.

32. Zhang $\mathrm{C}$, Xie L, Liang $\mathrm{H}$, Cui Y. LncRNA MIAT facilitates osteosarcoma progression by regulating mir128-3p/VEGFC axis. IUBMB Life. 2019;71(7):845-53.

33. Jiang $N$, Zhang $X, H e Y$, Luo B, He C, Liang Y, et al. Identification of key protein-coding genes and IncRNAs in spontaneous neutrophil apoptosis. Sci Rep. 2019;9(1):15106.

34. Shetab Boushehri MA, Lamprecht A. TLR4-Based Immunotherapeutics in Cancer: A Review of the Achievements and Shortcomings. Mol Pharm. 2018;15(11):4777-800.

35. Zhu K, Zhu X, Sun S, Yang W, Liu S, Tang Z, et al. Inhibition of TLR4 prevents hippocampal hypoxicischemic injury by regulating ferroptosis in neonatal rats. Exp Neurol. 2021;345:113828.

36. Wang J, Li Z, Gao A, Wen Q, Sun Y. The prognostic landscape of tumor-infiltrating immune cells in cervical cancer. Biomed Pharmacother. 2019;120:109444.

37. Khalil DN, Smith EL, Brentjens RJ, Wolchok JD. The future of cancer treatment: immunomodulation, CARs and combination immunotherapy. Nature reviews Clinical oncology. 2016;13(6):394.

38. Zou W, Wolchok JD, Chen L. PD-L1 (B7-H1) and PD-1 pathway blockade for cancer therapy: Mechanisms, response biomarkers, and combinations. Science translational medicine. 2016;8(328):328rv4.

39. Deng L, Liang H, Xu M, Yang X, Burnette B, Arina A, et al. STING-Dependent Cytosolic DNA Sensing Promotes Radiation-Induced Type I Interferon-Dependent Antitumor Immunity in Immunogenic Tumors. Immunity. 2014;41(5):843-52.

40. Deng L, Liang H, Burnette B, Beckett M, Darga T, Weichselbaum RR, et al. Irradiation and anti-PD-L1 treatment synergistically promote antitumor immunity in mice. J Clin Investig. 2014;124(2):687-95.

41. Drijvers JM, Gillis JE, Muijlwijk T, Nguyen TH, Gaudiano EF, Harris IS, et al. Pharmacologic Screening Identifies Metabolic Vulnerabilities of CD8(+) T Cells. Cancer immunology research. 2021;9(2):18499.

42. Tang R, Xu J, Zhang B, Liu J, Liang C, Hua J, et al. Ferroptosis, necroptosis, and pyroptosis in anticancer immunity. J Hematol Oncol. 2020;13(1):110.

43. Jiang Z, Lim SO, Yan M, Hsu JL, Yao J, Wei Y, et al. TYRO3 induces anti-PD-1/PD-L1 therapy resistance by limiting innate immunity and tumoral ferroptosis. The Journal of clinical investigation. 2021;131(8).

44. Zhou N, Bao J. FerrDb: a manually curated resource for regulators and markers of ferroptosis and ferroptosis-disease associations. Database: the journal of biological databases and curation. 2020;2020.

45. Tibshirani R. The lasso method for variable selection in the Cox model. Statistics in medicine. 1997;16(4):385-95. 
46. Simon N, Friedman J, Hastie T, Tibshirani R. Regularization Paths for Cox's Proportional Hazards Model via Coordinate Descent. Journal of statistical software. 2011;39(5):1-13.

47. Kanehisa M, Goto S. KEGG: kyoto encyclopedia of genes and genomes. Nucleic acids research. 2000;28(1):27-30.

48. Langfelder $P$, Horvath S. WGCNA: an R package for weighted correlation network analysis. BMC Bioinform. 2008;9:559.

49. Ringnér M. What is principal component analysis? Nature biotechnology. 2008;26(3):303-4.

50. Ho WJ, Jaffee EM, Zheng L. The tumour microenvironment in pancreatic cancer - clinical challenges and opportunities. Nature reviews Clinical oncology. 2020;17(9):527-40.

51. Yi M, Nissley DV, McCormick F, Stephens RM. ssGSEA score-based Ras dependency indexes derived from gene expression data reveal potential Ras addiction mechanisms with possible clinical implications. Sci Rep. 2020;10(1):10258.

52. Jiang P, Gu S, Pan D, Fu J, Sahu A, Hu X, et al. Signatures of T cell dysfunction and exclusion predict cancer immunotherapy response. Nature medicine. 2018;24(10):1550-8.

\section{Figures}




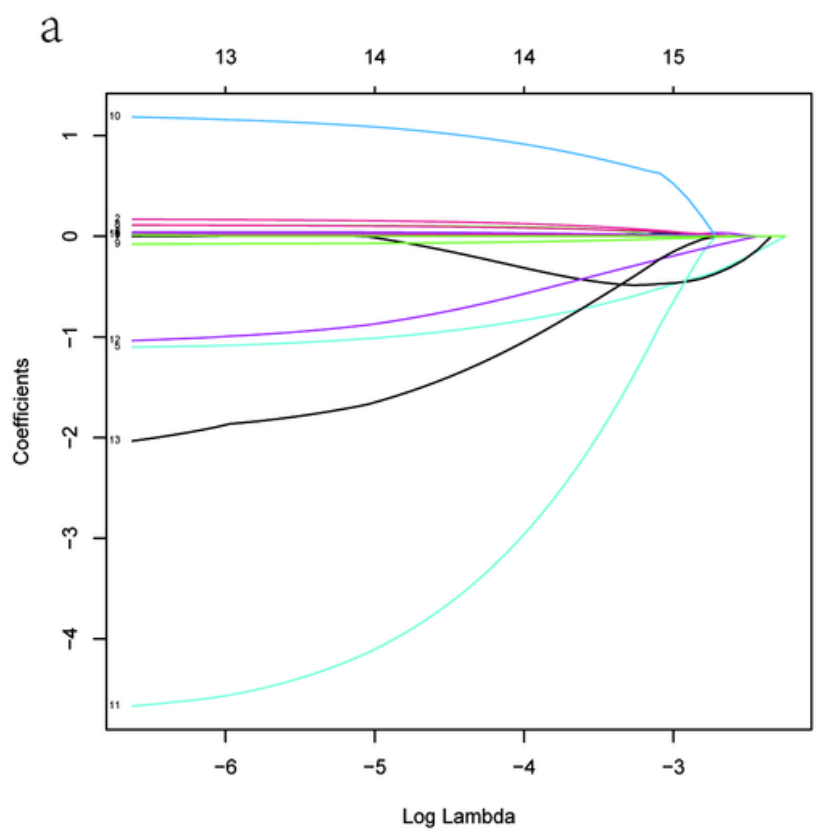

$\mathrm{b}$

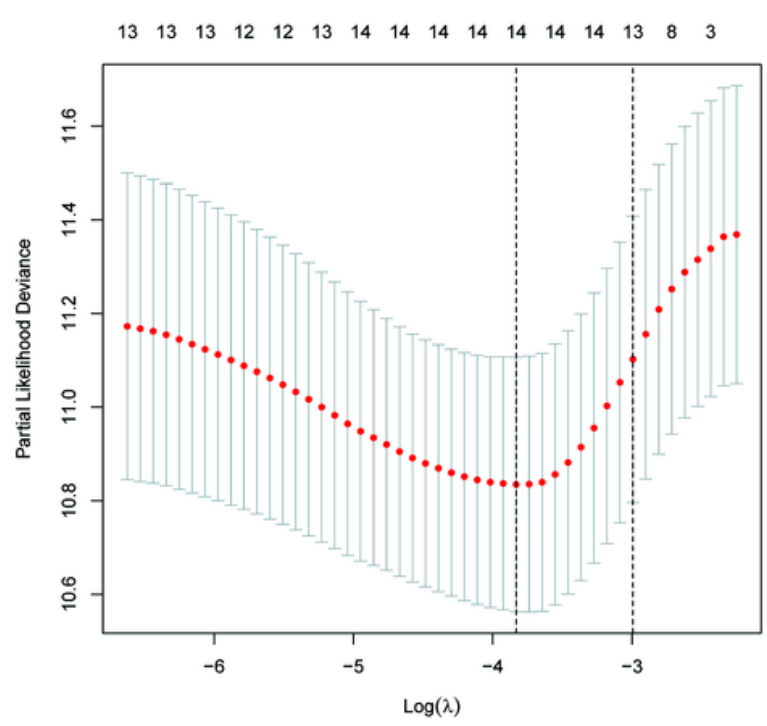

C

d
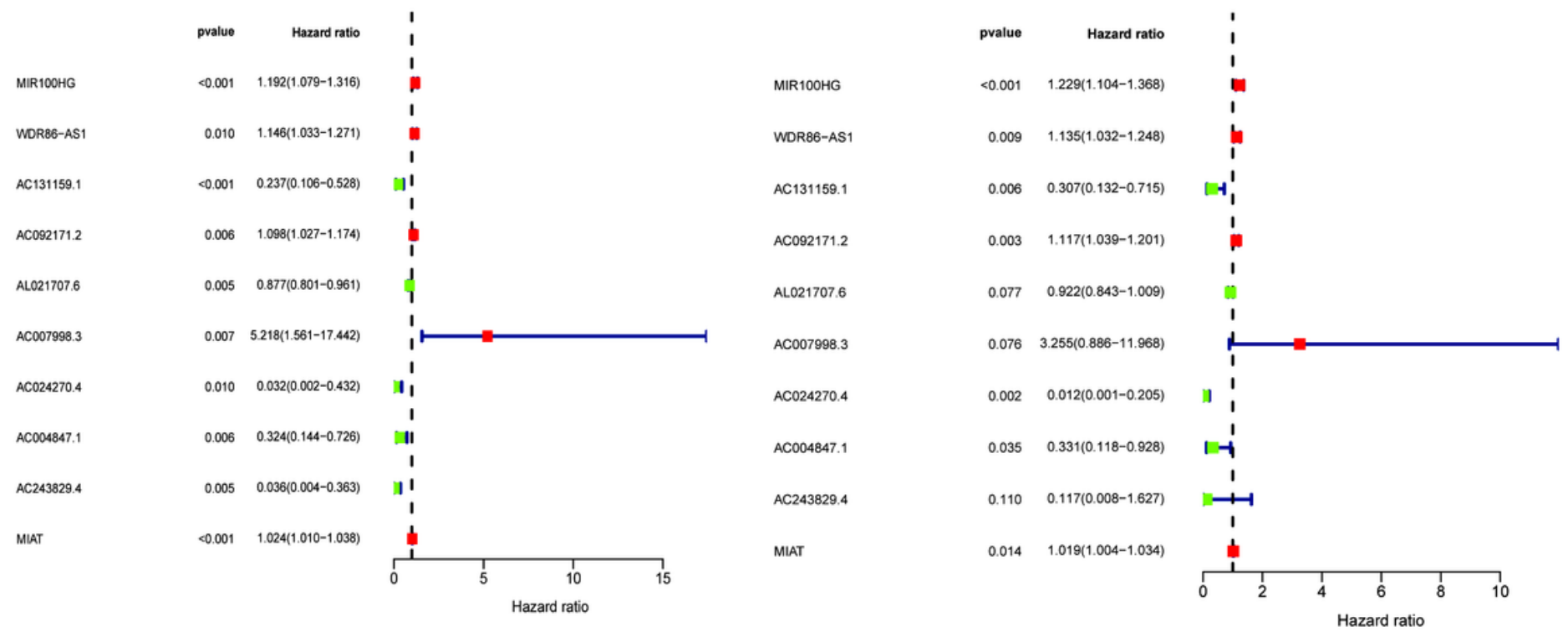

Figure 1

construction of ferroptosis-related IncRNA signature. (a) The coefficient of IncRNAs in CC (b) tuning parameter $(\lambda)$ based on cross-validation in FRLS (cd) Univariate and Multivariate Cox regression analysis indicated the relationship between 10 ferroptosis-related IncRNAs and OS 
a

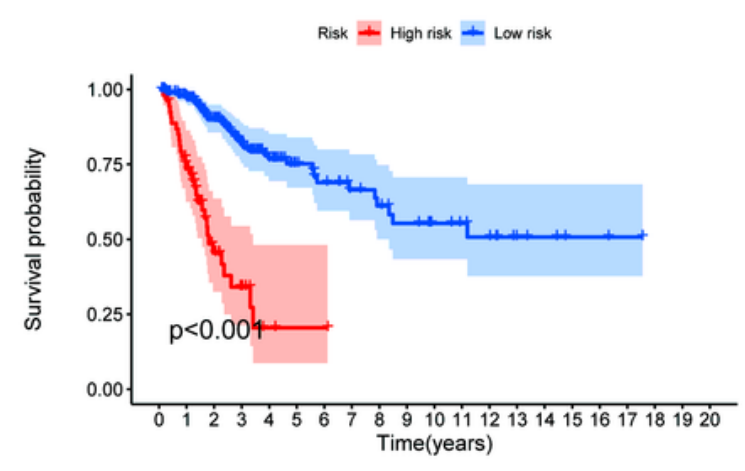

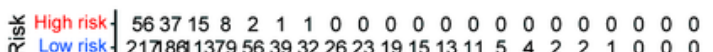

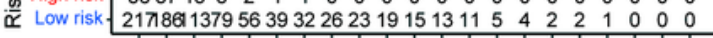

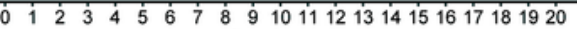
Time(years)

C

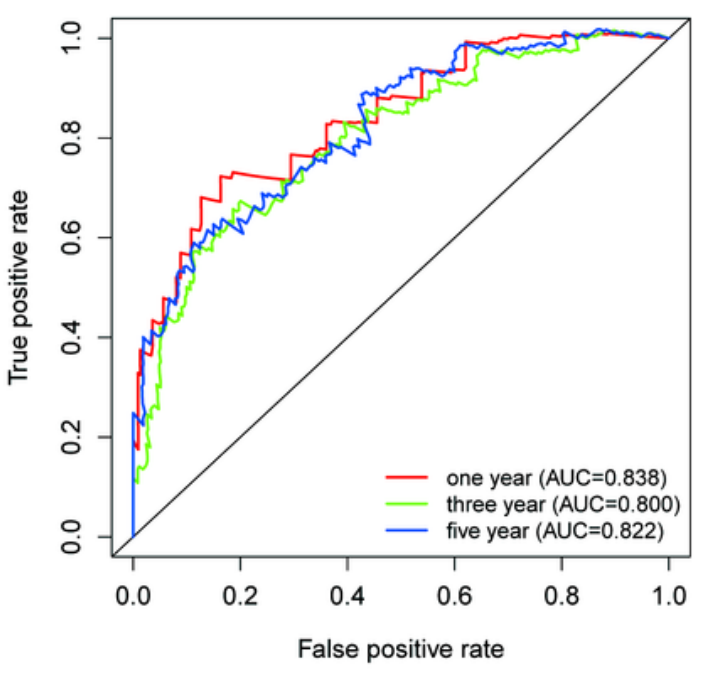

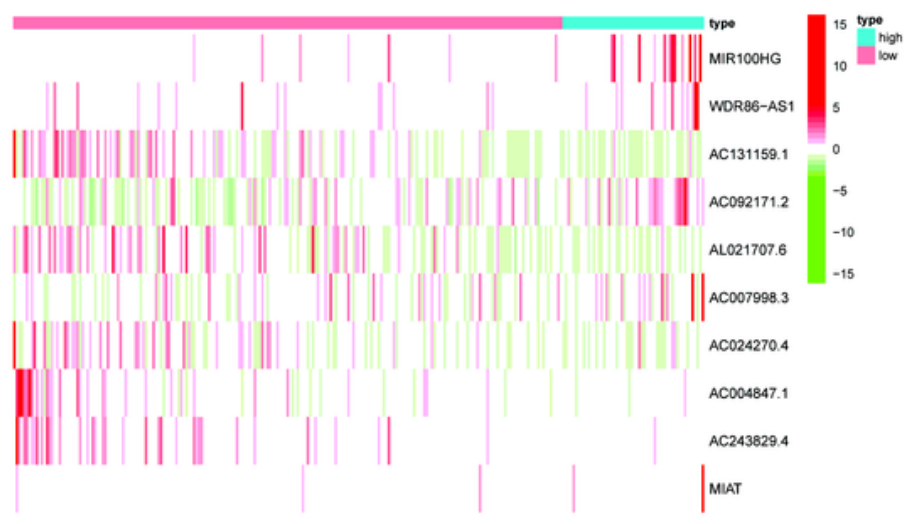

d

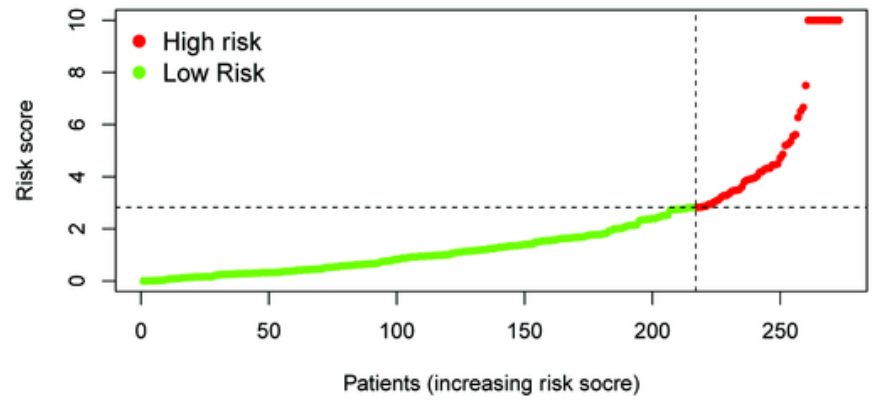

e

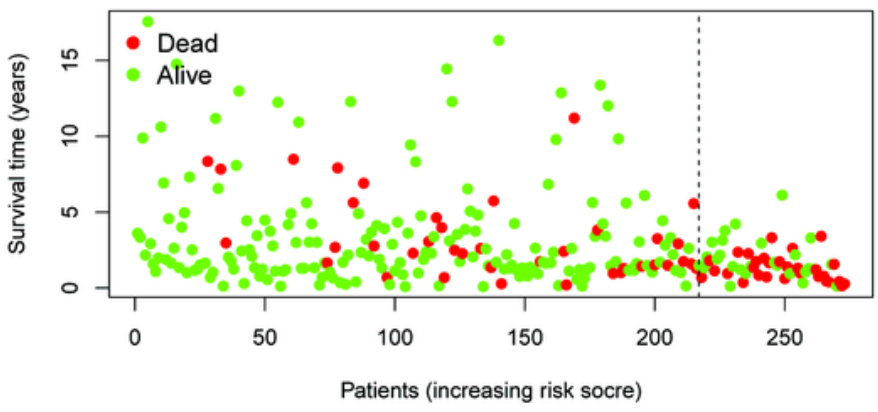

Figure 2

Evaluation of FRLS prognostic value. (a) Kaplan-Meier curve showed the correlation of the OS in low- and high-risk groups (b)The heatmap revealed the expression of 10 IncRNA for each patient (c) The Timedependent ROC of 1,3,5-year to evaluate the risk score of the signature The distribution and optimal cutoff value of (d) the risk scores and (e) the survival time in the TCGA database 


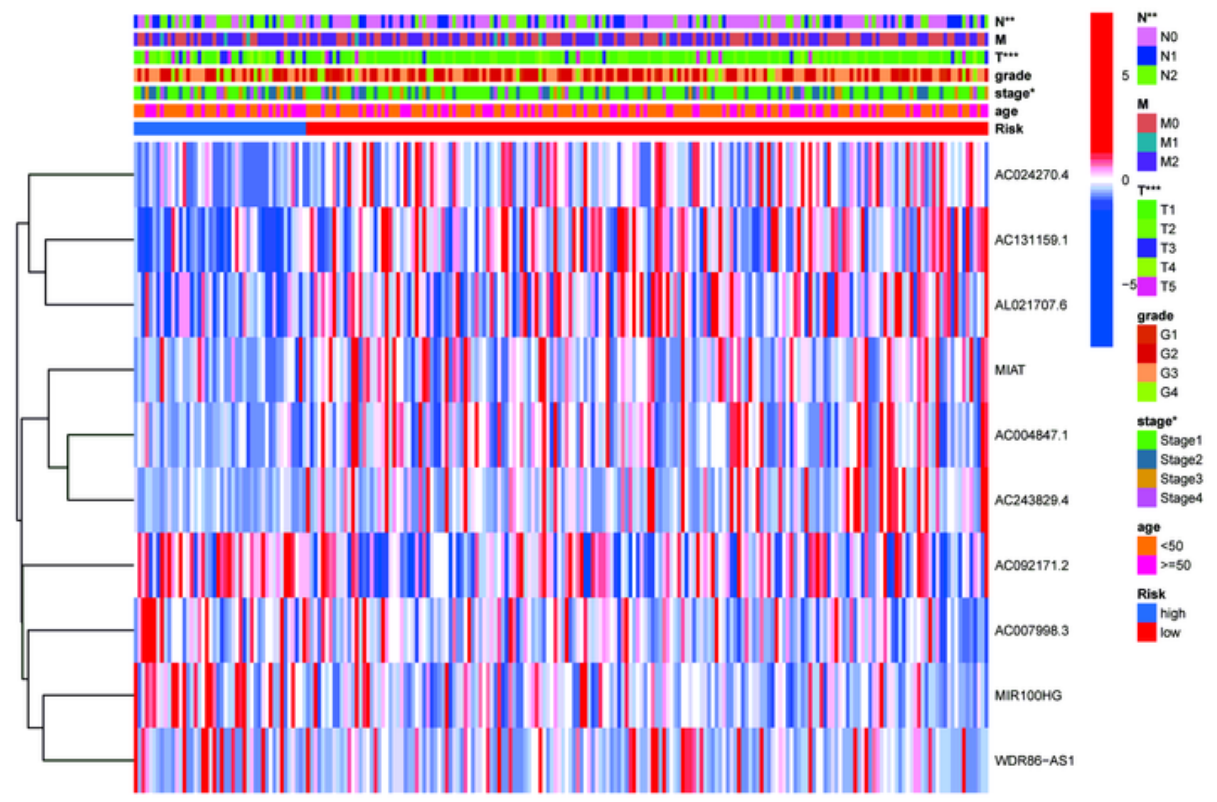

b

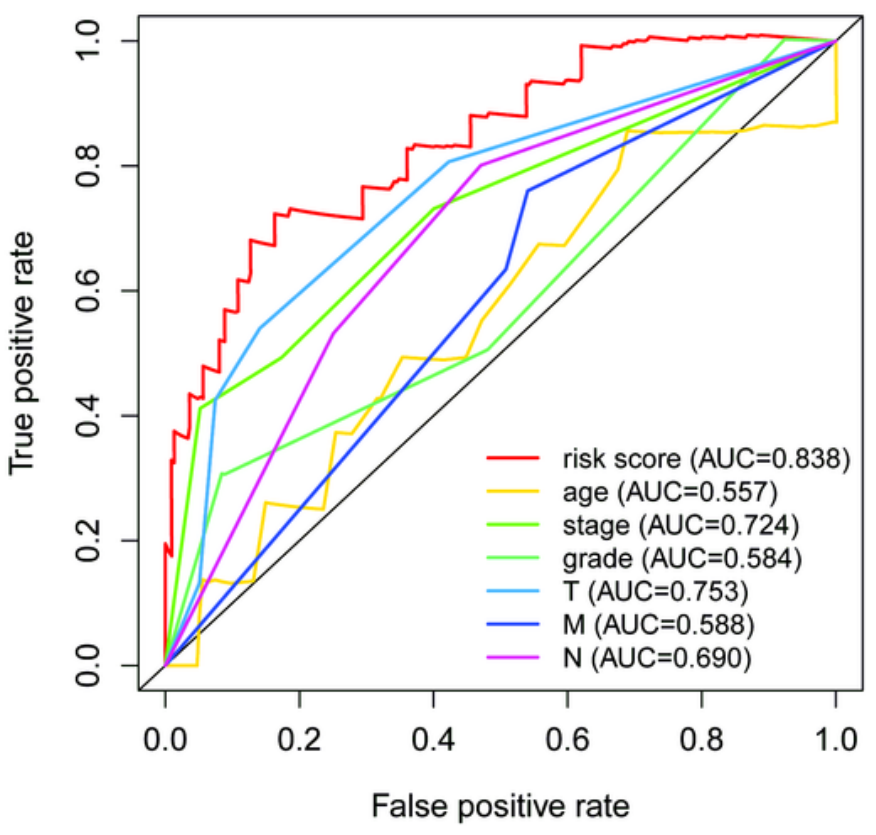

Figure 3

The relationship between clinicopathological characteristics and risk score (a)Heatmap for 10 ferroptosis-related IncRNAs and clinicopathological feature (b)ROC cure of risk factors and risk score 
a

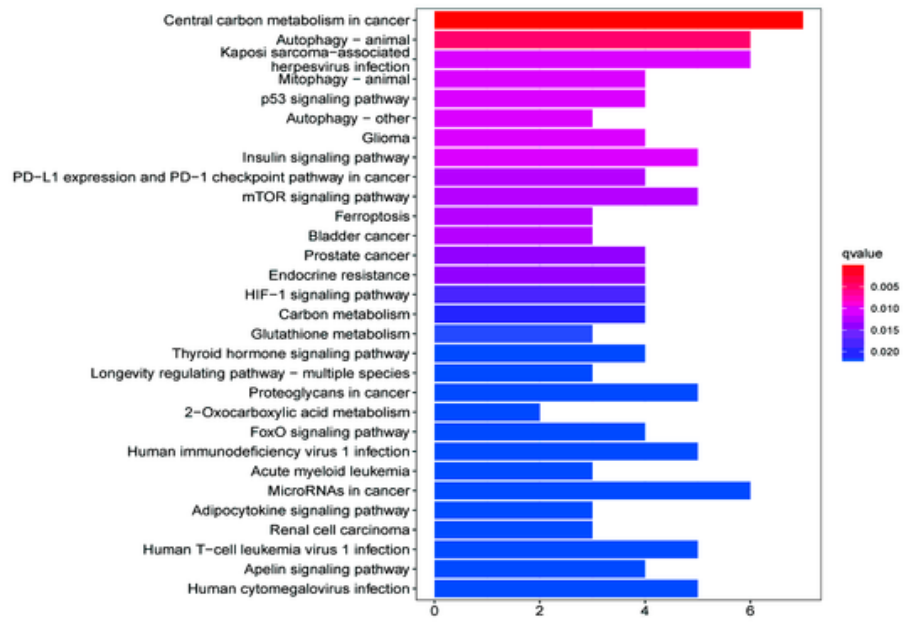

$\mathrm{b}$

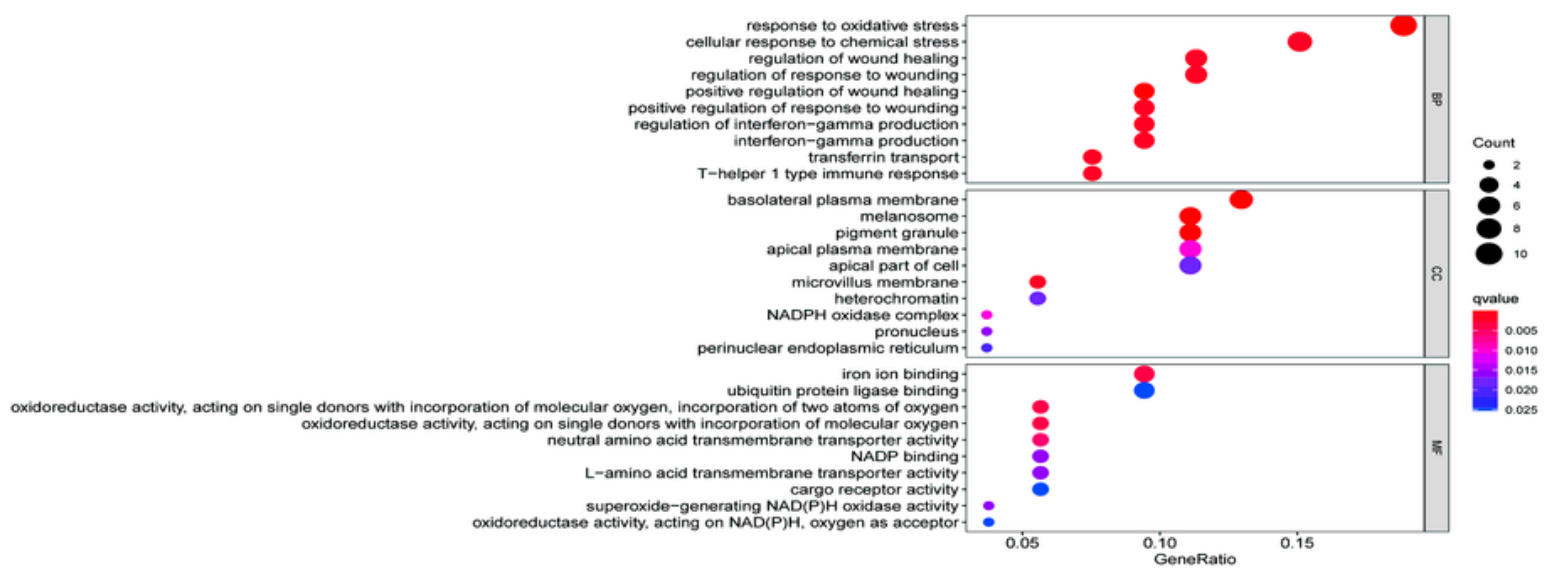

C
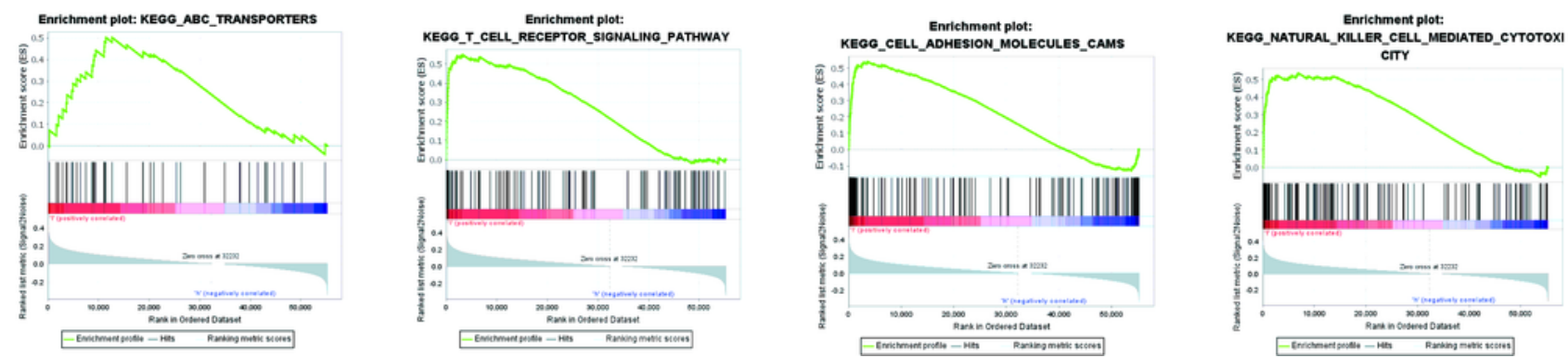

Figure 4

Enrichment analysis of differential expressed ferroptosis genes (a)GO (b)KEGG (c)GSEA of FRLS showed significant enrichment of the immune-related pathways 

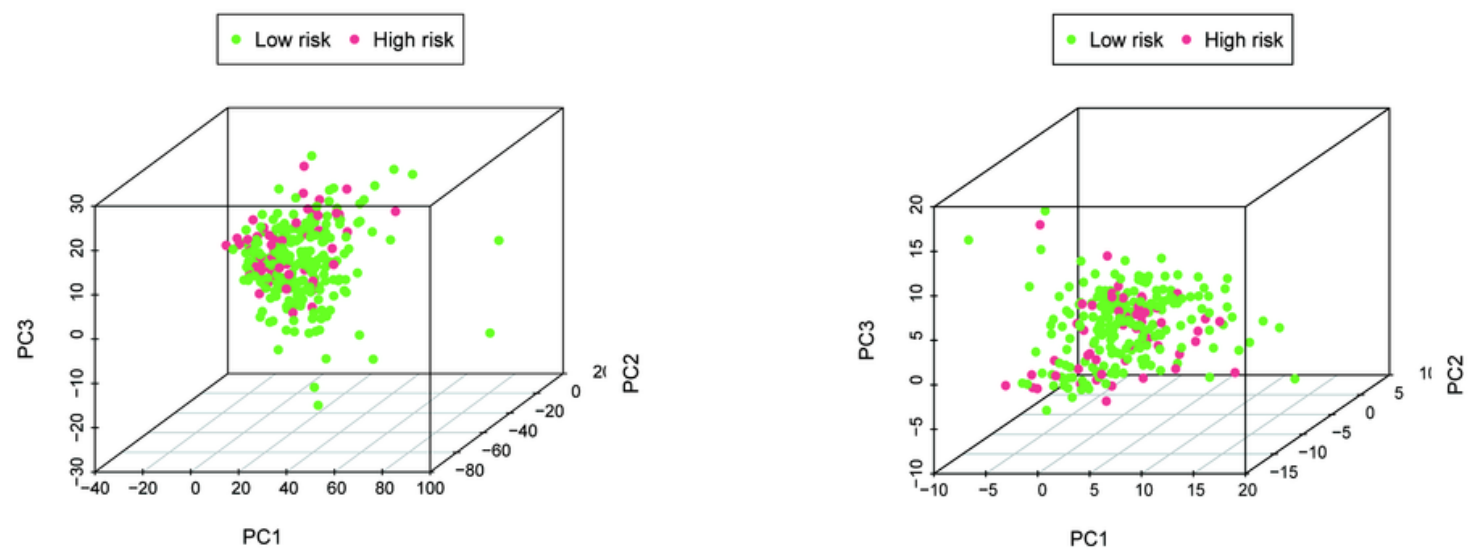

$\mathrm{C}$

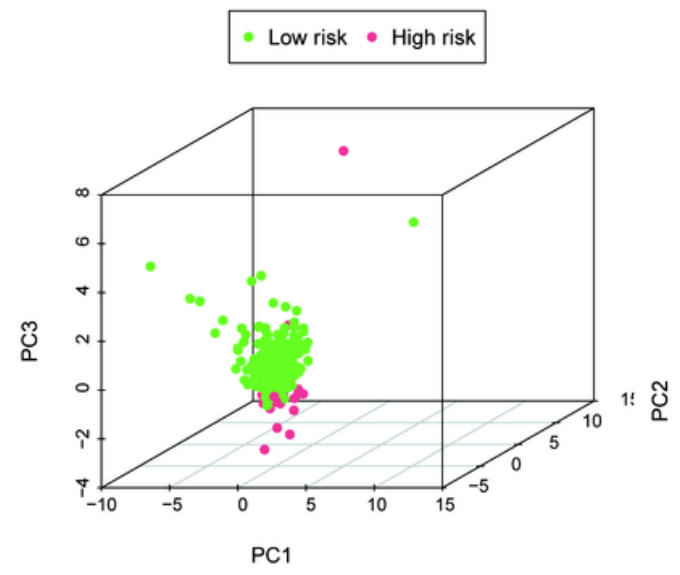

Figure 5

Principal component analysis(a) all IncRNAs (b) 512 ferroptosis-related IncRNAs (c) 10 IncRNAs based on FRLS between high- and low-risk groups 


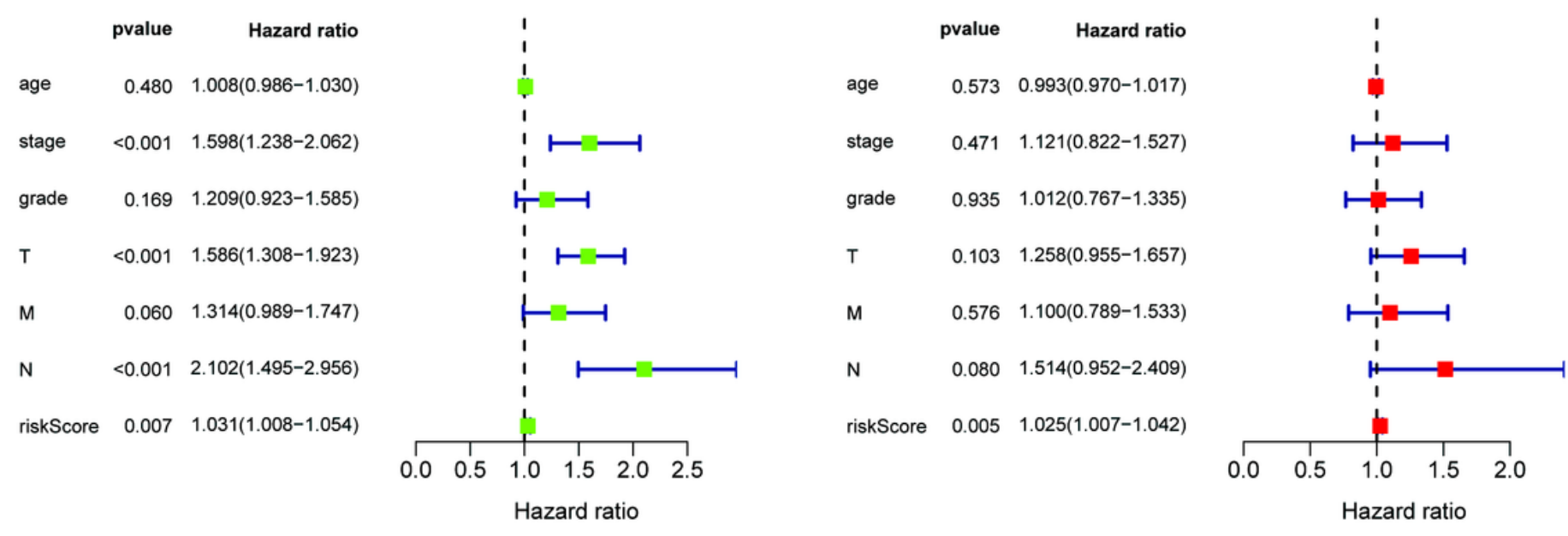

C

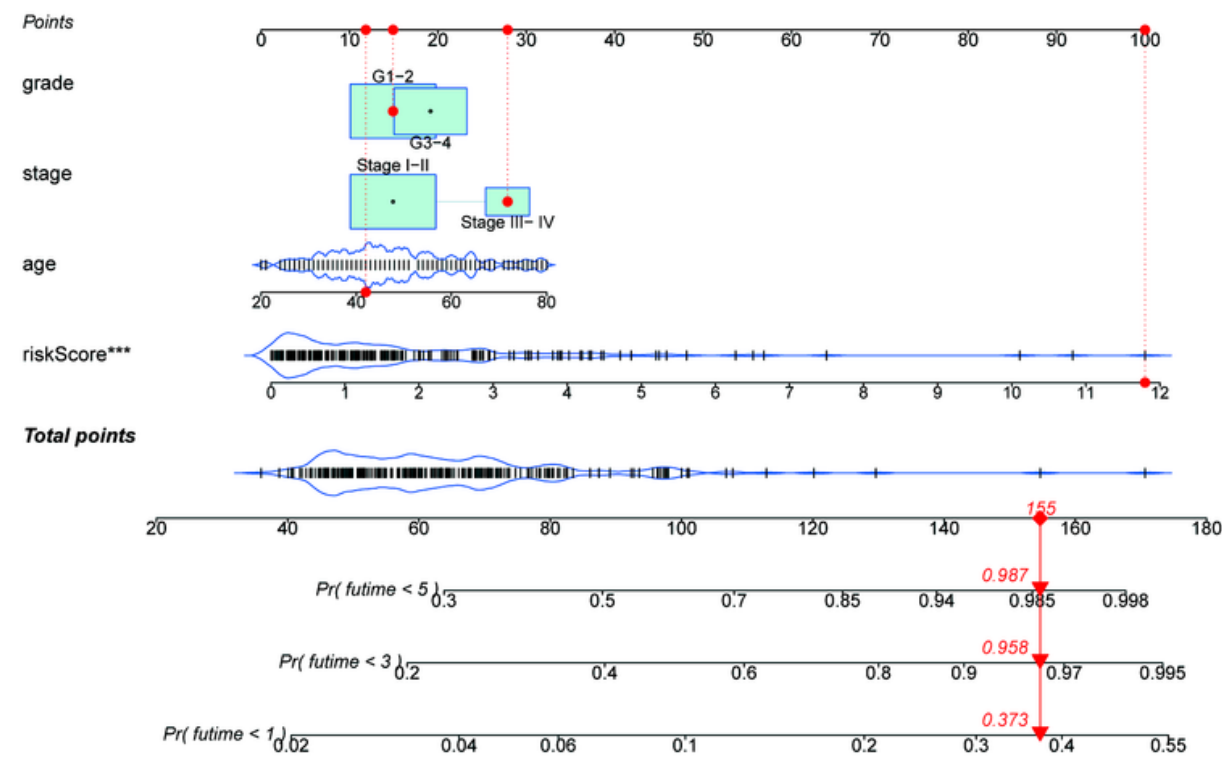

Figure 6

evaluation of the prognostic independence of FRLS (ab) Univariate and multivariate analysed the risk factors and riskscore (c)a nomogram for clinical risk factors and riskscore to predict 1,3,5-year OS 


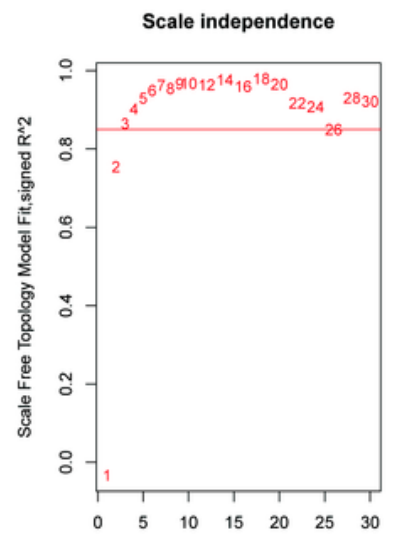

Soft Threshold (power)

$\mathrm{b}$

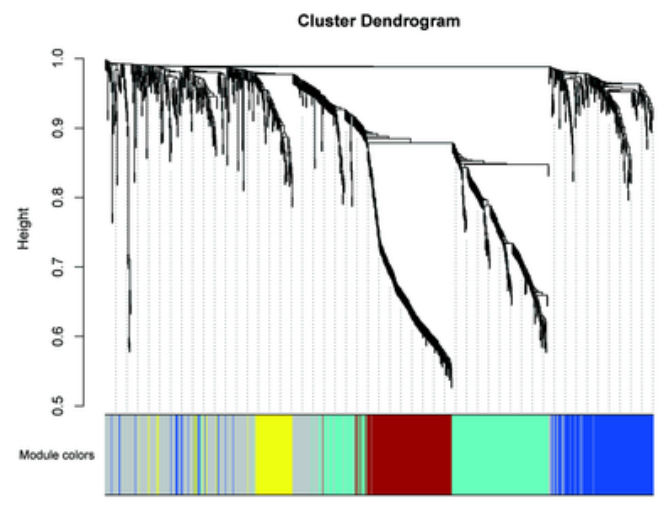

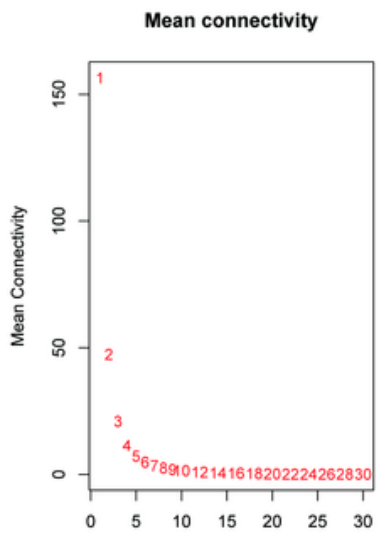

Soft Threshold (power)

乙

d
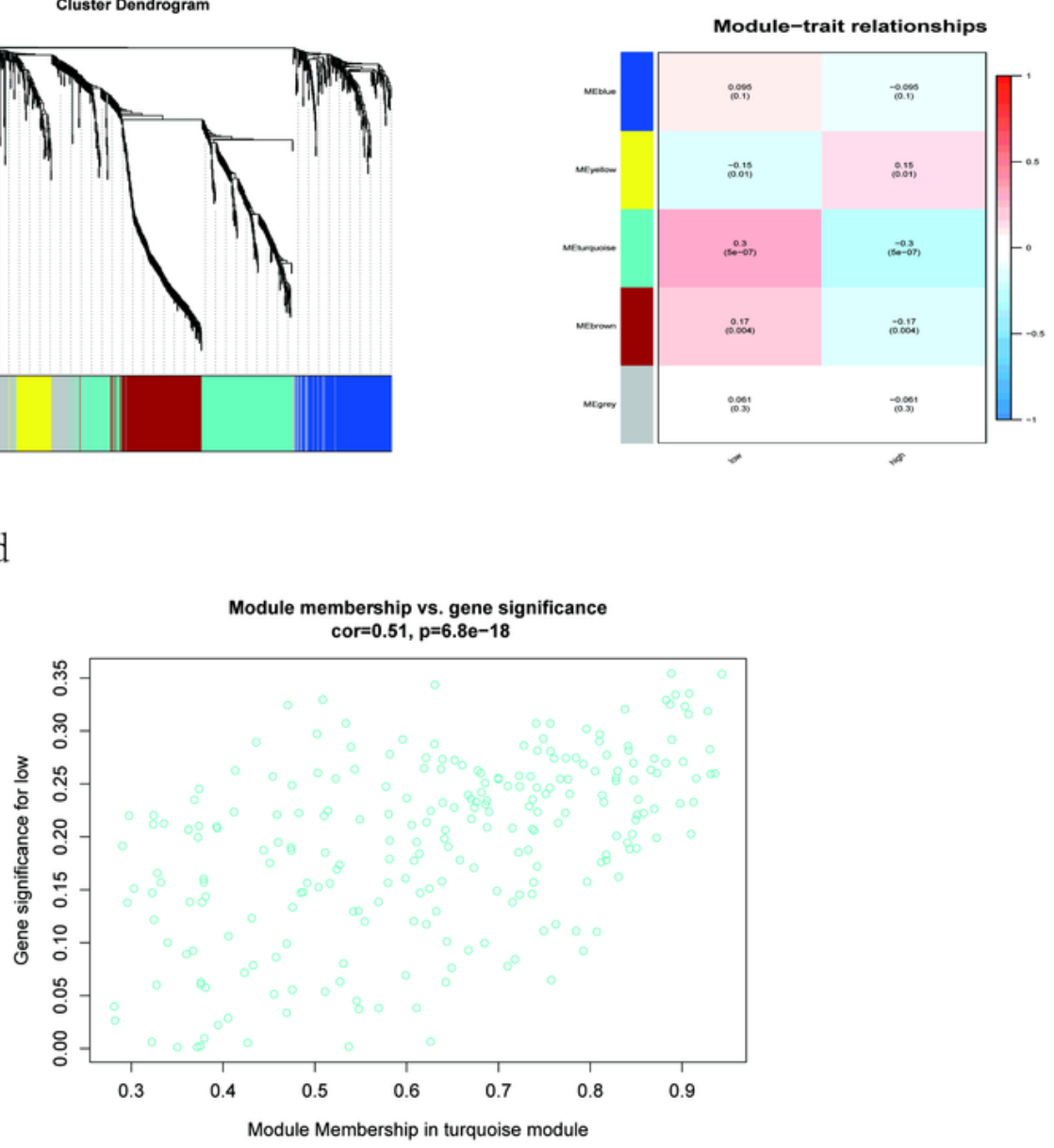

\section{Figure 7}

(a) Identification of soft threshold power $(\beta)$ of WGCNA $(\beta=3)$ (b) Hierarchical clustering dendrogram of immune genes clustered based on the measurement of dissimilarity (1-TOM). A total of 5 color modules, each containing a set of highly connected genes. (c) Identification of the optimal module (a) heatmap of the correlation between Eigengene and the risk (d) scatter plot of turquoise module (cor $=0.51$ ) 

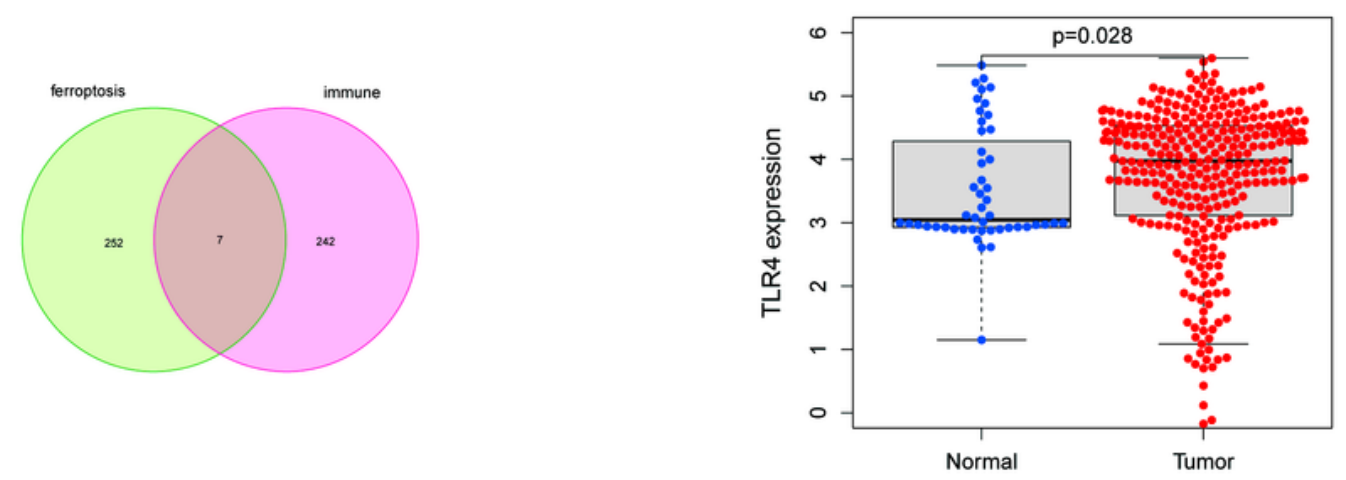

C
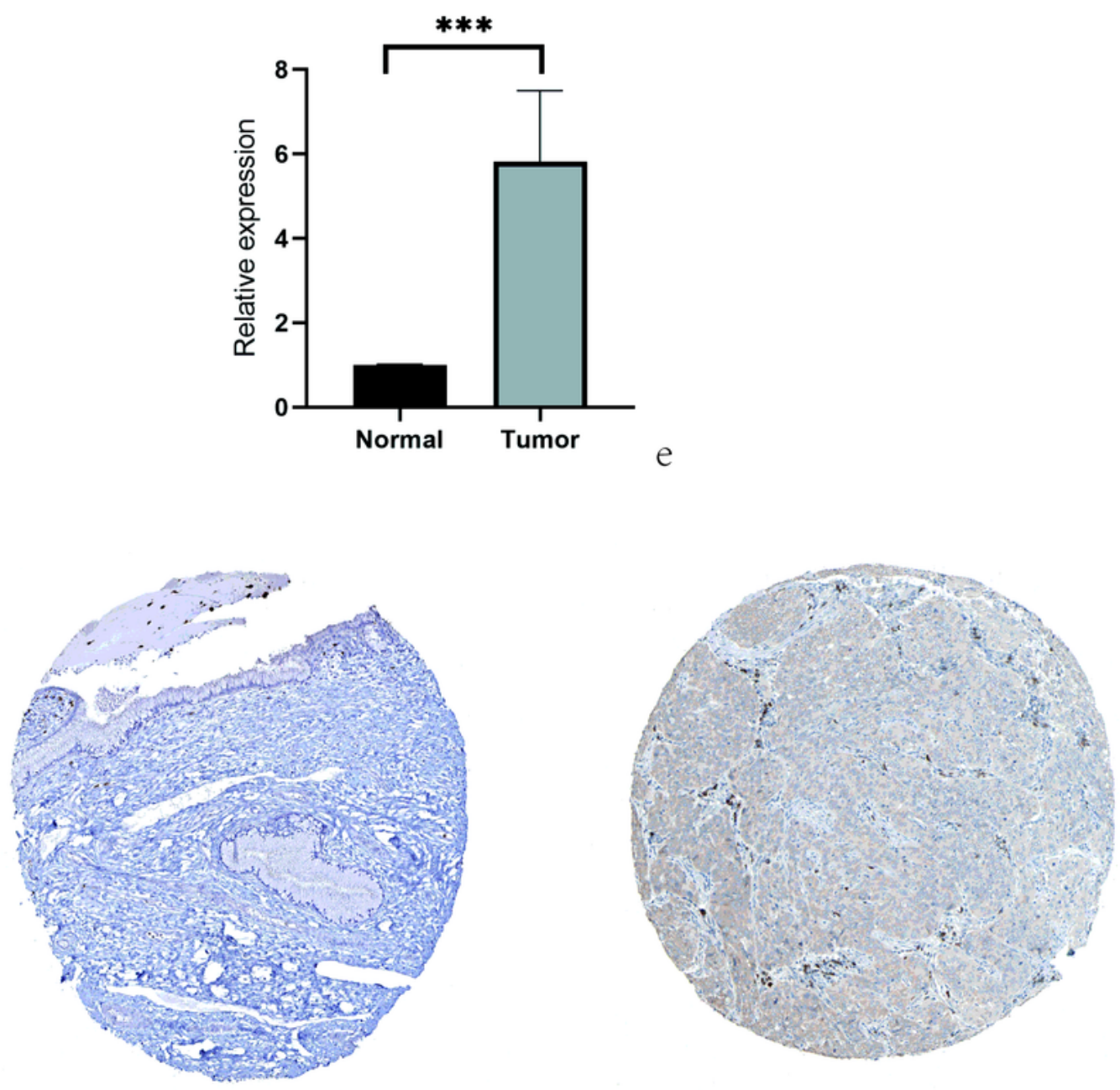

Figure 8

Identification and validation of gene (a) venn diagram of the intersection of ferroptosis genes and turquoise module genes (b) relative expression level of TLR4 in normal and tumor tissues of GSE9750, GSE44001, and GSE63514 datasets TLR4 protein level expression in the HPA database (c) immunohistochemistry of normal cervical tissue (d) immunohistochemistry of CC tissue. (e) Validation the relative expression of TLR4 via qRT-PCR 


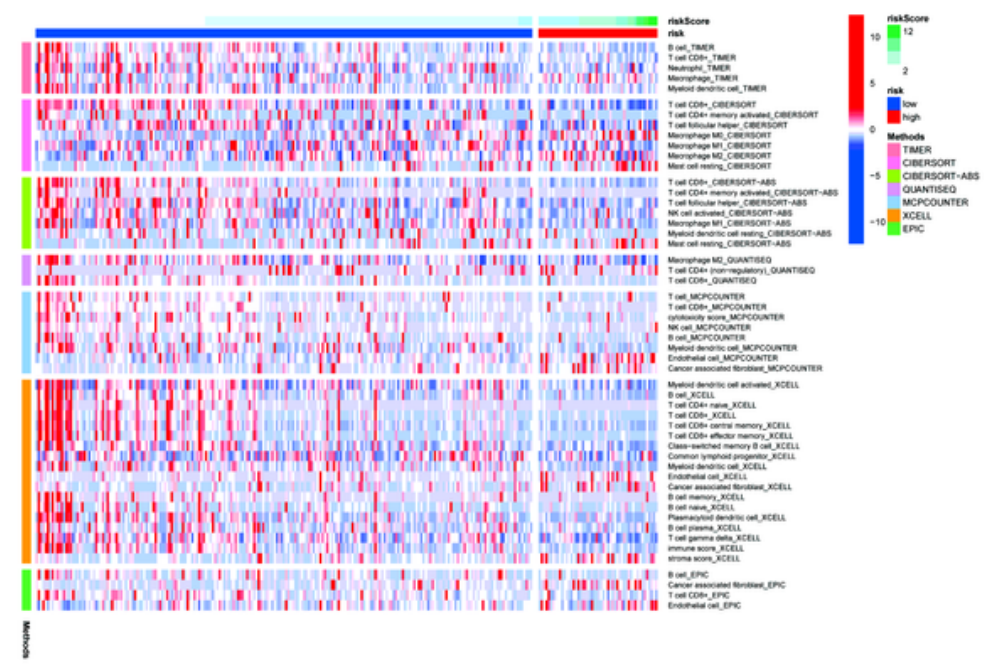

b

d
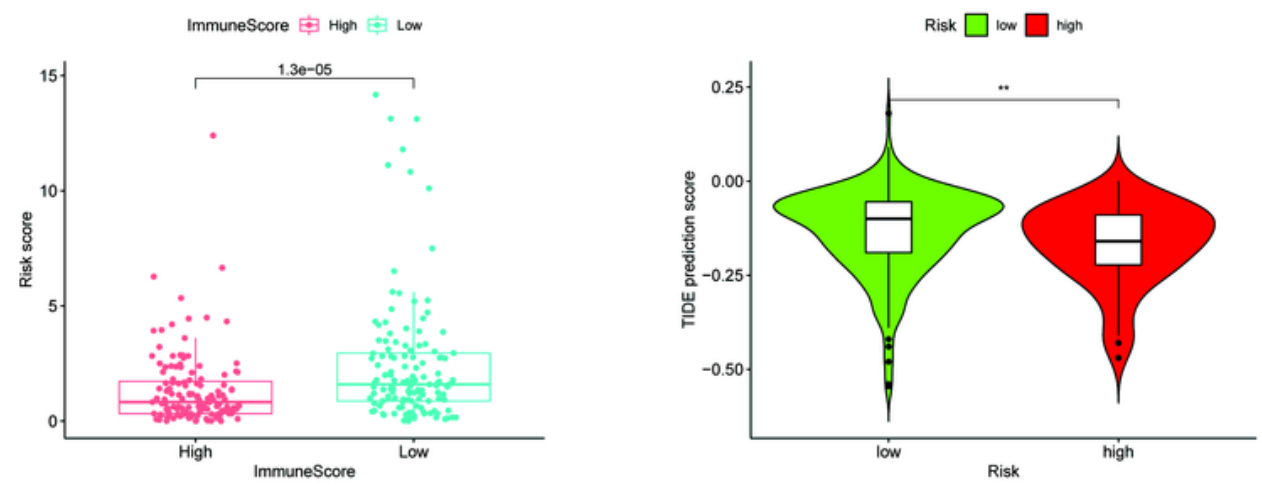

C

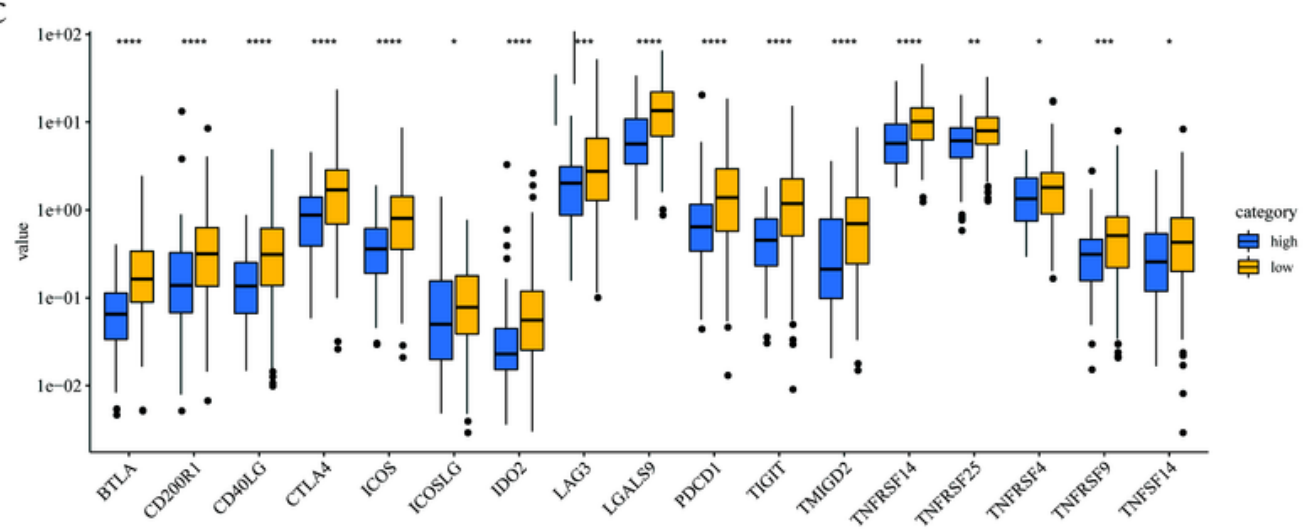

\section{Figure 9}

(a) Heatmap for immune cells based on multi algorithms among high- and low-risk groups (b)The difference of immunescore between low- and high-risk groups (c)The expression of immune checkpoints in low- and high-risk groups (d)TIDE score to predict the effectiveness of ICls treatment in low- and highrisk patients 


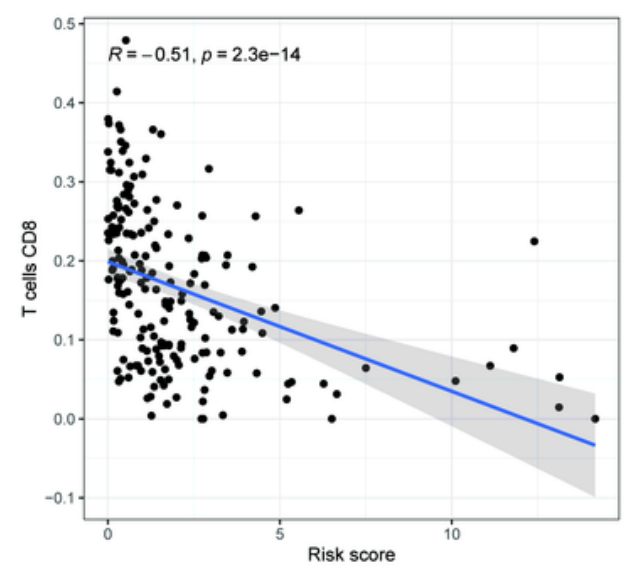

C

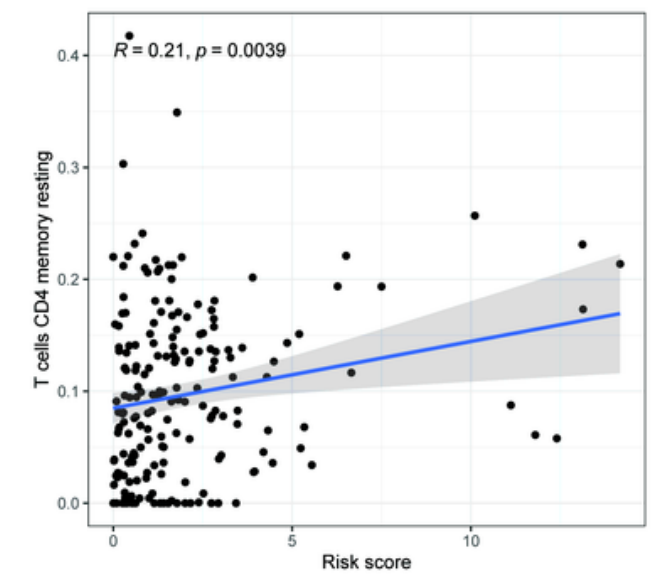

e

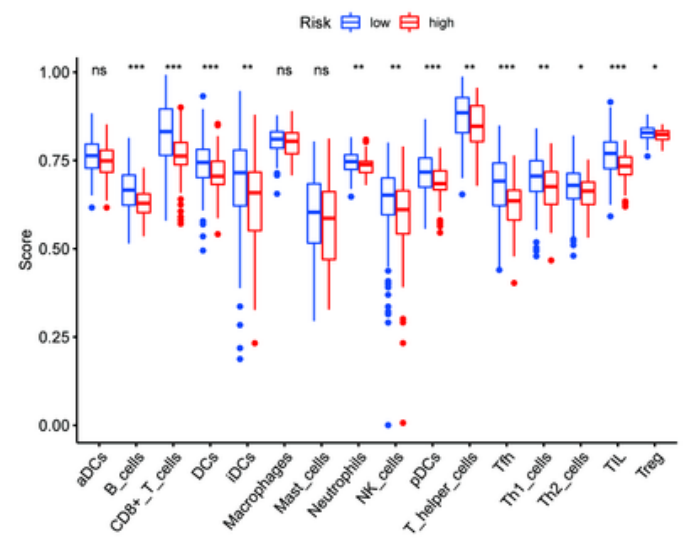

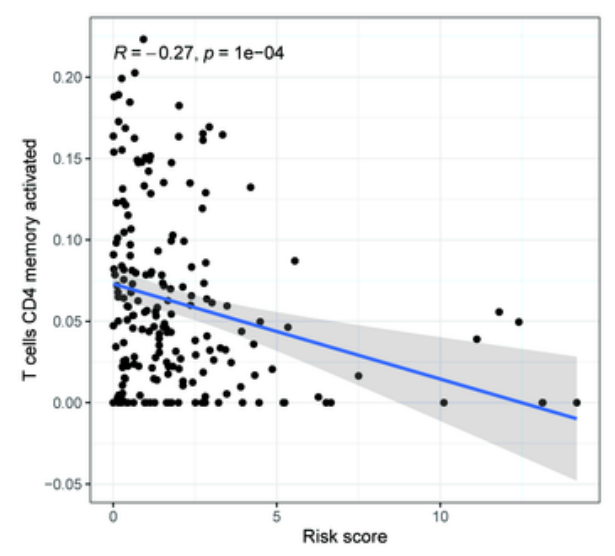

d

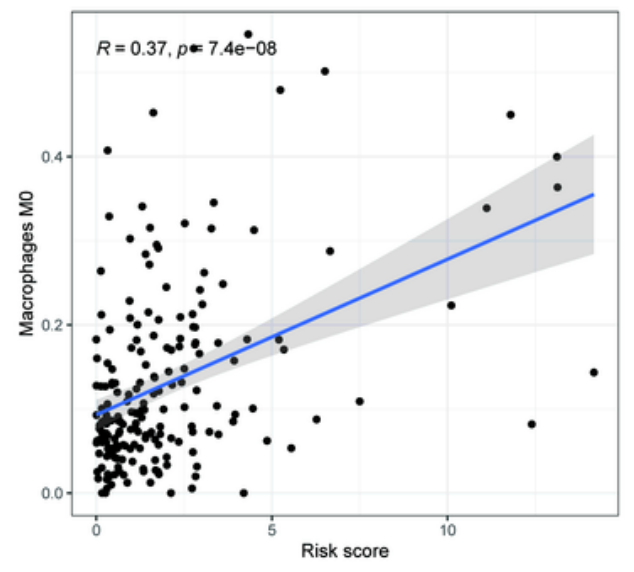

f

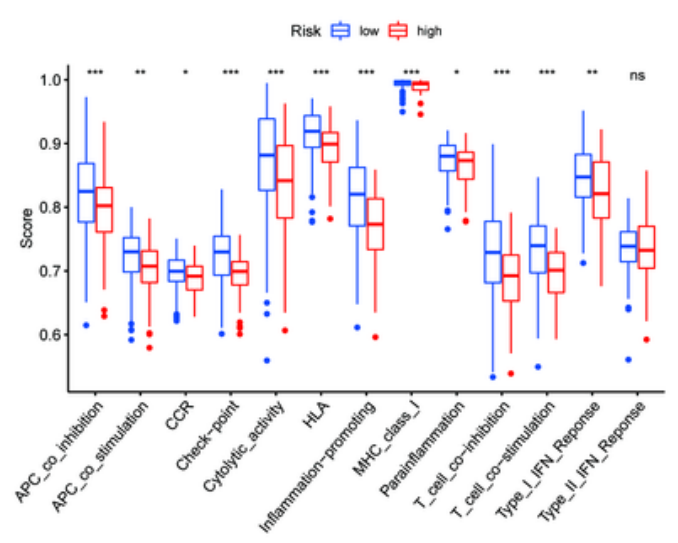

Figure 10

Correlation analysis of immune cells and the risk score(a)CD8 T cell(b) CD4 memory activation T-cell (c) CD4 memory resting T cells (d) Macrophages MO ssGSEA enrichment score of (e) immune cell (f)related function

\section{Supplementary Files}


This is a list of supplementary files associated with this preprint. Click to download.

- S1.tif 